\title{
Comparative accuracies of electrodiagnostic and imaging studies in neonatal brachial plexus palsy
}

\author{
Brandon W. Smith, MD, ${ }^{1}$ Kate W. C. Chang, MA, MS, ${ }^{1}$ Lynda J. S. Yang, MD, PhD, ${ }^{1}$ and \\ Mary Catherine Spires, PT, MS, MD² \\ Departments of ${ }^{1}$ Neurosurgery and ${ }^{2}$ Physical Medicine and Rehabilitation, University of Michigan, Ann Arbor, Michigan
}

\begin{abstract}
OBJECTIVE The incorporation of ancillary testing in the preoperative setting for patients with neonatal brachial plexus palsy (NBPP) remains controversial, but the recommendation for early nerve reconstruction when a baby has a preganglionic lesion at the lower nerve roots is generally accepted. At some specialty centers, nerve surgeons use preoperative electrodiagnostic testing (EDX) and imaging to aid in lesion localization and the preoperative planning of the nerve reconstruction. EDX and imaging have been evaluated for their abilities to detect pre- and postganglionic lesions, but their accuracies have never been compared directly in the same set of patients. The aim of the present study was to evaluate the accuracy of imaging and EDX in an NBPP population.
\end{abstract}

METHODS A retrospective review was conducted of 54 patients with operative NBPP seen between 2007 and 2017. The patients underwent EDX and imaging: EDX was performed, and the results were reviewed by board-certified electrodiagnosticians, and imaging was reviewed by board-certified neuroradiologists. The gold standard was considered to be the findings at surgical exploration. Descriptive and analytical statistics were utilized to compare the accuracies of imaging and EDX.

RESULTS The mean age at surgery was 6.94 mos ( \pm 4 mos). Fifteen patients (28\%) were Narakas grade I-II, and 39 $(72 \%)$ were Narakas grade III-IV. For all nerve roots, the overall accuracy of detecting preganglionic lesions was $74 \%$ for EDX and $69 \%$ for imaging. The overall sensitivity of detecting preganglionic lesions by EDX was $31 \%$, but the specificity was $90 \%$. The overall sensitivity of detecting preganglionic lesions by imaging was $66 \%$, and the overall specificity was $70 \%$. However, at C8, EDX was $37.5 \%$ sensitive and $87.5 \%$ specific, whereas imaging was $67.7 \%$ sensitive but only $29.4 \%$ specific.

CONCLUSIONS EDX outperformed imaging with regard to specificity and accuracy of identifying preganglionic injuries. This finding is especially relevant in the lower nerve roots, given that lower plexus preganglionic lesions are an accepted indication for early intervention.

https://thejns.org/doi/abs/10.3171/2018.7.PEDS18193

KEYWORDS neonatal brachial plexus palsy; imaging; electrodiagnostic testing; ganglionic lesion; avulsion injury; preoperative testing; peripheral nerve

$\mathrm{T}$ HE evaluation and treatment of neonatal brachial plexus palsy (NBPP) have continued to evolve and improve over the past 30 years. However, many controversial topics remain in this field. Across the major brachial plexus clinical programs, a wide variation persists in algorithms and decision making, specifically regarding the timing of surgery, indications, procedure selection, and the incorporation of ancillary testing. Preoperative ancillary testing may include electrodiagnostic testing (EDX) and various imaging modalities. The main goals of preoperative testing are to aid in the localization of the injury and assist in preoperative planning for nerve reconstruction. The utilization of preoperative testing remains controversial, with some centers performing both imaging and EDX and others avoiding the tests altogether.

One surgical indication that is not controversial is early intervention for avulsion injuries to the lower trunk..$^{10}$ Outcome studies have repeatedly shown a strongly posi-

ABBREVIATIONS EDX = electrodiagnostic testing; EMG = electromyography; NBPP = neonatal brachial plexus palsy; US = ultrasound.

SUBMITTED April 5, 2018. ACCEPTED July 18, 2018.

INCLUDE WHEN CITING Published online October 5, 2018; DOI: 10.3171/2018.7.PEDS18193. 
tive benefit for children who undergo early repair. The widespread acceptance of this indication demonstrates the importance of accurately identifying avulsion injuries in the preoperative setting. Diagnostic imaging includes both CT myelography and MRI myelography, which have approximately $60 \%-80 \%$ accuracy in identifying avulsion injuries. ${ }^{5,12,16}$ EDX demonstrates a low sensitivity but relatively high specificity in detecting avulsion injury, $19 \%$ and $93 \%$, respectively. A difficulty arises when both preganglionic and postganglionic injuries are present, as this can lead to inaccurate labeling of the injury as an isolated postganglionic lesion. Because electrodiagnostics and overall imaging have not been compared in a headto-head fashion in the same patients, the goal of this study was to evaluate the comparative accuracy of EDX and imaging in the detection of neonatal brachial plexus avulsions in the same patient.

\section{Methods \\ Study Design}

This was a retrospective review of 54 infants with operative NBPP treated between 2007 and 2017 in a single institute. We included consecutive infants who underwent nerve surgery with preoperative imaging (CT, MRI, or ultrasound [US]) and EDX. The current protocol involves diagnostic imaging (first-line MRI) at 3 months if no signs of recovery exist, and electrodiagnostics at 1 month or at the time of referral. Patient demographics (age, sex, and race) were reported, and NBPP-specific variables, such as involved side and the Narakas grade, were evaluated in an interdisciplinary NBPP clinic. The Narakas grade was determined by a single surgeon based on either 1) physical examination and neurological assessment at initial clinical appointment or approximately 1 month of age, or 2) mother's or obstetrician's report of infant's hand/ arm movements at birth. The preoperative imaging and EDX accuracies of NBPP lesion detection (preganglionic and postganglionic) were verified by the surgical findings (gold standard) at the root and trunk levels. We reported sensitivity, specificity, and accuracy of EDX and imaging compared to surgical findings to evaluate the performance accuracy of ancillary studies. The study protocol was approved by the institutional review board.

\section{Preoperative EDX and Imaging \\ Electrodiagnostic Criteria}

Physicians who were board certified by the American Board of Electrodiagnostic Medicine performed the electrodiagnostic testing on all infants prior to nerve reconstruction surgery. Motor and sensory median and ulnar nerve conduction studies were performed on the involved upper extremity. The ulnar sensory nerve response was recorded from the small finger (5th digit), while the median sensory responses were recorded from the middle finger (3rd digit) and either the thumb or index finger. Collected nerve conduction study data included amplitude, distal latency, and conduction velocity.

Using a pediatric concentric needle, electromyography (EMG) examination was performed on relevant muscle sets in the outpatient setting without sedation. The EMG data collected included insertional activity, presence or absence of spontaneous activity (positive waves, fibrillation, and fasciculation potentials), motor unit action potential numbers, and morphology (polyphasia, motor unit frequency, and the size and number of motor units).

A lesion was determined to be preganglionic (avulsion) if the sensory nerve conduction studies were normal or greater than $50 \%$ of normal compared to the uninvolved upper extremity or laboratory norms and EMG abnormalities were present in the correlated weak/flaccid muscles. The lesions were determined to be postganglionic (rupture) if the sensory conductions studies were abnormali.e., the nerve conduction studies of the involved limb were less than $50 \%$ of the normal limb or laboratory norms and EMG abnormalities were present in the relevant muscles.

\section{Imaging Criteria}

The imaging of the brachial plexus was protocoled and evaluated by board-certified pediatric neuroradiologists. The imaging options included both CT myelography and MR myelography, and discussion with the radiologist prior to the image acquisition determined the modality and sequencing. CT myelography was primarily used prior to the development of the MR myelography protocol. Now MR myelography is our first-line choice. These imaging modalities require general anesthesia. Direct evaluation for the presence of ventral nerve root continuity was utilized to determine the presence of an avulsion injury; the presence of a pseudomeningocele was not considered diagnostic. The specific protocols have been detailed by Somashekar et al. ${ }^{12,13}$

\section{Statistical Analysis}

We applied descriptive statistics for patient demographic and surgical findings. Regarding the accuracy of detecting preganglionic and postganglionic lesions, we report sensitivity (true positive/[true positive + false negative]), specificity (true negative/[false positive + true negative]), and accuracy (true positive + true negative/all) of both EDX and imaging at root (C5-T1) and trunk (upper, middle, and lower) levels.

\section{Results}

Fifty-four patients were included in this study. The mean age at initial appointment was 2.67 months $( \pm 3$ months), and the mean age at surgery was 6.94 months ( \pm 4 months). Thirty-one patients (57\%) were females and 34 (63\%) were Caucasian. The right upper extremity was involved in 30 patients (56\%). There were 15 (28\%) Narakas grade I-II patients and 39 (72\%) Narakas grade III-IV patients. Forty-four patients (81\%) underwent imaging: MRI + US, 27 (50\%); CT only, 13 (24\%); MRI only, 2 (4\%); and CT + US + MRI, 2 (4\%) (Table 1). The injuries were determined at surgery and varied by nerve root level (Table 2).

The detection of an avulsion by EDX is detailed by root level in Table 3. The overall sensitivity was 31\% (range $14 \%-50 \%$ ), with higher sensitivity at lower root levels; the overall specificity was $90 \%$ (range 78\%-96\%), and total accuracy was $74 \%$ (range $71 \%-78 \%$ ). The detection of avulsions by imaging is detailed by root level in Table 3 . 
TABLE 1. Summary of demographic data for 54 patients

\begin{tabular}{cc}
\hline \multicolumn{1}{c}{ Variable } & Value \\
\hline Mean age \pm SD & \\
\hline At initial appointment (mos) & $2.67 \pm 3$ \\
\hline At surgery (mos) & $6.94 \pm 4$ \\
\hline Sex & $23(43 \%)$ \\
\hline Male & $31(57 \%)$ \\
\hline Female & \\
\hline Race & $34(63 \%)$ \\
\hline Caucasian & $20(37 \%)$ \\
\hline Other & $24(44 \%)$ \\
\hline NBPP-involved side & $30(56 \%)$ \\
\hline Left & $15(28 \%)$ \\
\hline Right & $39(72 \%)$ \\
\hline Narakas grade & \\
\hline I-II & $27(50 \%)$ \\
\hline III-IV & $13(24 \%)$ \\
\hline Imaging type & $10(19 \%)$ \\
\hline MRI + US & $2(4 \%)$ \\
\hline CT only & $2(4 \%)$ \\
\hline None & \\
\hline MRI only & \\
\hline CT + US + MRI & \\
\hline
\end{tabular}

Values are number of patients (\%) unless otherwise indicated.

The overall sensitivity was $66 \%$ (range $42 \%-100 \%$ ), overall specificity was $70 \%$ (range $29 \%-85 \%$ ), and total accuracy was $69 \%$ (range $43 \%-77 \%$ ).

The EDX detection of an avulsion injury when grouped by trunk is detailed in Table 4 . The sensitivity ranged from $30 \%$ to $41.7 \%$, specificity ranged from $83.3 \%$ to $92.3 \%$, and accuracy ranged from $71 \%$ to $75 \%$. The imaging detection of avulsions grouped by trunk is detailed in Table 4. The sensitivity ranged from $53.3 \%$ to $83.3 \%$, specificity ranged from $41.9 \%$ to $84.2 \%$, and accuracy ranged from $53 \%$ to $76 \%$.

The EDX detection of rupture at each root level is detailed in Table 5. The overall sensitivity was $90 \%$ (range $78 \%-96 \%$ ), overall specificity was $31 \%$ (range $14 \%-50 \%$ ), and total accuracy was $74 \%$ (range $71 \%-78 \%$ ). The imaging detection of rupture is detailed by root level in Table 5 . The overall sensitivity was $70 \%$ (range $29 \%-85 \%$ ), overall specificity was $66 \%$ (range $42 \%-100 \%$ ), and total accuracy was $69 \%$ (range $43 \%-77 \%$ ). The sensitivities of detecting a rupture by utilizing EDX in the upper, middle, and lower trunks were $91.7 \%, 92.3 \%$, and $83.3 \%$, respectively (Table 6); the specificities in the upper, middle, and lower trunks were $33 \%, 30 \%$, and $41.7 \%$, respectively. The accuracy in detecting rupture using EDX was $75 \%$ in both the upper and middle trunks and $71 \%$ in the lower trunk. The sensitivities of detecting a rupture by utilizing imaging in the upper, middle, and lower trunks were $84.2 \%, 73.3 \%$, and $41.9 \%$, respectively (Table 6); the specificities of detecting a rupture in the upper, middle, and lower trunk were $53.3 \%, 83.3 \%$, and $41.9 \%$, respectively. The accuracy in de-
TABLE 2. Summary of injuries by nerve root in 54 patients

\begin{tabular}{lccccc}
\hline \multicolumn{1}{c}{ Injury } & C5 & C6 & C7 & C8 & T1 \\
\hline Rupture & $47(87 \%)$ & $30(56 \%)$ & $41(76 \%)$ & $29(54 \%)$ & $25(46 \%)$ \\
\hline Avulsion & $7(13 \%)$ & $24(44 \%)$ & $12(22 \%)$ & $9(17 \%)$ & $5(9 \%)$ \\
\hline Normal & - & - & $1(2 \%)$ & $8(15 \%)$ & $8(15 \%)$ \\
\hline Not applicable & - & - & - & $8(15 \%)$ & $16(30 \%)$ \\
\hline
\end{tabular}

$-=$ not applicable.

tecting rupture using imaging was $74 \%$ in the upper trunk, $76 \%$ in the middle trunk, and $53 \%$ in the lower trunk.

\section{Discussion}

Neonatal brachial plexus palsy occurs in 1-4 of every 1000 births in the United States. ${ }^{1,6}$ Fortunately, many of these patients recover, but $30 \%-40 \%$ are left with permanent deficits. ${ }^{8,11}$ The treatment of patients with persistent neurological deficits varies from center to center, but primary nerve surgery includes either nerve grafting or nerve transfer procedures. Although decision-making algorithms for surgical repair vary, serial physical examinations remain a standard at most centers. . $^{2,4,719}$ A detailed physical examination can localize the lesion by defining the affected muscle groups. However, the physical examination falls short in defining the underlying anatomy of the injury, especially whether a nerve rupture or a root avulsion is present. Currently, the one widely accepted indication for early intervention in NBPP is a pan-plexus injury with avulsion of the lower nerve roots. Consequently, in patients with hand weakness in the setting of a brachial plexus injury, it is of paramount importance to determine if an avulsion injury is present in the lower roots. Any improvements in our ability to identify an avulsion injury in the lower trunk would be a step forward in the care of these patients, since a clinical examination cannot easily predict this pathology.

The gold standard evaluation for the presence of rupture or avulsion is operative exploration, but preoperative knowledge of the anatomy of the injury could be helpful in planning the nerve reconstruction strategy and timing of intervention, as well as in prognostication. Ancillary tests such as imaging and EDX, in addition to the clinical examination, are utilized to determine this anatomy. Centers vary in the use of these tests, and ultimately their incorporation remains controversial.

The two most common imaging modalities for the detection of avulsion injuries are CT myelography and MR myelography. ${ }^{12-14} \mathrm{CT}$ myelography has been found to be $63 \%-72.2 \%$ sensitive in the detection of avulsion injuries in brachial plexus palsy. ${ }^{5,16}$ Findings on CT myelography, including pseudomeningoceles and the absence of nerve rootlets, are indicative of a rupture. The two major concerns when using CT myelography in NBPP are the lumbar puncture and the child's exposure to ionizing radiation. MR myelography is a newer modality that avoids both the invasiveness of the lumbar puncture and exposure to ionizing radiation. ${ }^{12}$ Recent studies have demonstrated an overall sensitivity of $75 \%$ and a specificity of $83 \%$ for 
TABLE 3. Sensitivity and specificity of EDX and imaging detection of avulsion by nerve root

\begin{tabular}{cccccccc}
\hline & \multicolumn{3}{c}{ EDX (no. of patients) } & & \multicolumn{2}{c}{ Imaging (no. of patients) } \\
\cline { 2 - 3 } \cline { 7 - 8 } Nerve Root & Sensitivity & Specificity & Accuracy & & Sensitivity & Specificity & Accuracy \\
\hline C5 & $14.3 \%(1$ of 7$)$ & $88.4 \%(38$ of 43$)$ & $78 \%(39$ of 50$)$ & & $42.9 \%(3$ of 7$)$ & $83.8 \%(31$ of 37$)$ & $77 \%(34$ of 44$)$ \\
\hline C6 & $39.1 \%(9$ of 23$)$ & $96.6 \%(28$ of 29$)$ & $71 \%(37$ of 52$)$ & & $56.5 \%(13$ of 23$)$ & $85 \%(17$ of 20$)$ & $70 \%(30$ of 43$)$ \\
\hline C7 & $30 \%(3$ of 10$)$ & $92.3 \%(24$ of 26$)$ & $75 \%(27$ of 36$)$ & & $83.3 \%(10$ of 12$)$ & $73.3 \%(22$ of 30$)$ & $76 \%(32$ of 42$)$ \\
\hline C8 & $37.5 \%(3$ of 8$)$ & $87.5 \%(14$ of 16$)$ & $71 \%(17$ of 24$)$ & & $66.7 \%(6$ of 9$)$ & $29.4 \%(5$ of 17$)$ & $43 \%(11$ of 26$)$ \\
\hline T1 & $50 \%(2$ of 4$)$ & $78.6 \%(11$ of 14$)$ & $72 \%(13$ of 18$)$ & & $100 \%(5$ of 5$)$ & $57.1 \%(8$ of 14$)$ & $68 \%(13$ of 19$)$ \\
\hline Total & $31 \%(18$ of 52$)$ & $90 \%(115$ of 128$)$ & $74 \%(133$ of 180$)$ & $66 \%(37$ of 56$)$ & $70 \%(83$ of 118$)$ & $69 \%(120$ of 174$)$ \\
\hline
\end{tabular}

TABLE 4. Sensitivity and specificity of EDX and imaging detection of avulsion by trunk

\begin{tabular}{|c|c|c|c|c|c|c|}
\hline \multirow[b]{2}{*}{ Nerve Trunk } & \multicolumn{3}{|c|}{ EDX (no. of patients) } & \multicolumn{3}{|c|}{ Imaging (no. of patients) } \\
\hline & Sensitivity & Specificity & Accuracy & Sensitivity & Specificity & Accuracy \\
\hline C5-6 & $33 \%(10$ of 30$)$ & $91.7 \%$ (66 of 72$)$ & $75 \%$ (76 of 102$)$ & $53.3 \%$ (16 of 30$)$ & $84.2 \%$ (48 of 57$)$ & $74 \%$ (64 of 87$)$ \\
\hline $\mathrm{C7}$ & $30 \%$ (3 of 10$)$ & $92.3 \%$ (24 of 26$)$ & $75 \%$ (27 of 36$)$ & $83.3 \%$ (10 of 12$)$ & $73.3 \%$ (22 of 30$)$ & $76 \%$ (32 of 42$)$ \\
\hline $\mathrm{C} 8-\mathrm{T} 1$ & $41.7 \%(5$ of 12$)$ & $83.3 \%(25$ of 30$)$ & $71 \%(30$ of 42$)$ & $78.6 \%$ (11 of 14$)$ & $41.9 \%$ (13 of 31$)$ & $53 \%(24$ of 45$)$ \\
\hline
\end{tabular}

TABLE 5. Sensitivity and specificity of EDX and imaging detection of rupture by root

\begin{tabular}{cccccccc}
\hline & \multicolumn{3}{c}{ EDX (no. of patients) } & & \multicolumn{2}{c}{ Imaging (no. of patients) } \\
\cline { 2 - 3 } \cline { 7 - 8 } Nerve Root & Sensitivity & Specificity & Accuracy & & Sensitivity & Specificity & Accuracy \\
\hline C5 & $88.4 \%(38$ of 43$)$ & $14.3 \%(1$ of 7$)$ & $78 \%(39$ of 50$)$ & & $83.8 \%(31$ of 37$)$ & $42.9 \%(3$ of 7$)$ & $77 \%(34$ of 44$)$ \\
\hline C6 & $96.6 \%(28$ of 29$)$ & $39.1 \%(9$ of 23$)$ & $71 \%(37$ of 52$)$ & & $85 \%(17$ of 20$)$ & $56.5 \%(13$ of 23$)$ & $70 \%(30$ of 43$)$ \\
\hline C7 & $92.3 \%(24$ of 26$)$ & $30 \%(3$ of 10$)$ & $75 \%(27$ of 36$)$ & & $73.3 \%(22$ of 30$)$ & $83.3 \%(10$ of 12$)$ & $76 \%(32$ of 42$)$ \\
\hline C8 & $87.5 \%(14$ of 16$)$ & $37.5 \%(3$ of 8$)$ & $71 \%(17$ of 24$)$ & & $29.4 \%(5$ of 17$)$ & $66.7 \%(6$ of 9$)$ & $43 \%(11$ of 26$)$ \\
\hline T1 & $78.6 \%(11$ of 14$)$ & $50 \%(2$ of 4$)$ & $72 \%(13$ of 18$)$ & & $57.1 \%(8$ of 14$)$ & $100 \%(5$ of 5$)$ & $68 \%(13$ of 19$)$ \\
\hline Total & $90 \%(115$ of 128$)$ & $31 \%(18$ of 52$)$ & $74 \%(133$ of 180$)$ & $70 \%(83$ of 118$)$ & $66 \%(37$ of 56$)$ & $69 \%(120$ of 174$)$ \\
\hline
\end{tabular}

TABLE 6. Sensitivity and specificity of EDX and imaging detection of rupture by trunk

\begin{tabular}{cccccccc}
\hline & \multicolumn{3}{c}{ EDX (no. of patients) } & & \multicolumn{3}{c}{ Imaging (no. of patients) } \\
\cline { 2 - 3 } Nerve Trunk & Sensitivity & Specificity & Accuracy & & Sensitivity & Specificity & Accuracy \\
\hline C5-6 & $91.7 \%(66$ of 72$)$ & $33 \%(10$ of 30$)$ & $75 \%(76$ of 102$)$ & & $84.2 \%(48$ of 57$)$ & $53.3 \%(16$ of 30$)$ & $74 \%(64$ of 87$)$ \\
\hline C7 & $92.3 \%(24$ of 26$)$ & $30 \%(3$ of 10$)$ & $75 \%(27$ of 36$)$ & & $73.3 \%(22$ of 30$)$ & $83.3 \%(10$ of 12$)$ & $76 \%(32$ of 42$)$ \\
\hline C8-T1 & $83.3 \%(25$ of 30$)$ & $41.7 \%(5$ of 12$)$ & $71 \%(30$ of 42$)$ & & $41.9 \%(13$ of 31$)$ & $78.6 \%(11$ of 14$)$ & $53 \%(24$ of 45$)$ \\
\hline
\end{tabular}

the identification of an avulsion injury with MR myelography. ${ }^{12}$ Our data demonstrated similar sensitivity and specificity for detecting avulsion injuries with imaging-70\% and $66 \%$, respectively. In 2018 , Wade et al. ${ }^{18}$ published a study focusing on the diagnostic accuracy of MRI in adult brachial plexus injuries. They found an overall diagnostic accuracy for root avulsions on MRI of $79 \%$, whereas the sole finding of a pseudomeningocele was $68 \%$. Our study found that the overall accuracy of imaging in detecting avulsion in NBPP was $69 \%$, but the overall accuracy of EDX in detecting avulsion injuries was $74 \%$.
Intraoperative electrodiagnostics, including somatosensory evoked potentials, are utilized to determine the anatomical localization of an injury. However, there is a lack of consensus for the use of preoperative electrodiagnostic testing. Electrodiagnostically, an avulsion injury is diagnosed in the presence of abnormal or absent compound motor action potentials associated with intact sensory nerve action potentials. The persistence of a sensory nerve action potential is due to the dorsal root ganglion housing the cell body of the sensory axons, which is distal to the injury site in root avulsions. A previous study demonstrat- 
ed that EDX was $19.5 \%$ sensitive and $93.2 \%$ specific in identifying avulsions; a similar study with follow-up demonstrated a sensitivity of $27.7 \%$ for detecting avulsions. ${ }^{15,16}$ Our data demonstrated an overall sensitivity of $31 \%$ but a specificity of $90 \%$ for detecting nerve root avulsions with the use of electrodiagnostics. Given this pattern of low sensitivity and high specificity, EDX appears to have more utility in verifying the likely presence of an avulsion. Due to the low sensitivity of EDX in detecting avulsions, it is best utilized as a confirmatory test rather than a screening test for detecting the presence of occult injuries that would be detected on physical examination and presentation. Preoperative EMG has been evaluated and was shown to overestimate recovery in the neonatal brachial plexus. As a result, most centers have moved away from incorporating this into their algorithms. ${ }^{3,8,17}$

Previous studies have examined the accuracy of CT myelography versus electrodiagnostics, ${ }^{15,16}$ but there is no evaluation of overall imaging versus EDX in the same patients. Some patients are referred with imaging prior to consultation, and the combination of both imaging modalities gives these data a broader application. Our data demonstrated that imaging was more sensitive than EDX in detecting an avulsion, $66 \%$ versus $31 \%$, respectively. However, in terms of overall specificity EDX outperformed imaging, $90 \%$ versus $70 \%$, respectively. The most marked differences were noted in the lower roots, with EDX being $87.5 \%$ specific at $\mathrm{C} 8$ and $78.6 \%$ specific at T1. Comparatively, the specificity of imaging in detecting avulsions in the lower root was $29.4 \%$ at C8 and $57.1 \%$ at T1. These data demonstrate that with regard to the lower roots, C8 and T1, EDX is a much more specific modality for detecting avulsions.

There is convincing evidence that patients with nerve root avulsions and lack of hand function require a surgical intervention, and these are generally accepted as indications for early intervention. ${ }^{10}$ In addition, the underlying anatomy of a nerve injury is important when choosing a reconstruction strategy. For instance, if the patient has an avulsion injury, then that nerve root would not be a candidate for nerve grafting. Imaging and EDX help to determine this preoperatively and when planning the likely repair strategy. The preoperative knowledge also helps us to counsel families on the reconstructive plan and the associated risks, which differ between graft repair and nerve transfer. Furthermore, there is also some evidence that earlier surgical intervention is beneficial in selected patients, although the exact timing of surgery remains controversial. Our data demonstrate that EDX is more accurate and more specific than imaging in identifying avulsion injuries, particularly in the lower nerve roots. The role for a highly specific test, like EDX, in patients with suspected lower brachial plexus injury would be to aid in identifying cases involving avulsion injuries. In summary, the utilization of EDX in the outpatient setting could help to identify lower plexus avulsions with a high specificity, potentially leading to earlier interventions and improved outcomes.

The first major limitation of this project is the relatively low number of patients included in the study, which makes drawing conclusions more difficult. However, with a rare presentation, we feel that these data are beneficial. This is a retrospective study, and the general limitations of retrospective analyses apply here. In relation to the retrospective nature of this project, not all patients had their initial evaluations done in our clinic, and a mixture of medical documentation and patient reporting was used to define the initial extent of injury. There is a heterogeneity of imaging types in our analysis; however, given that referrals can include imaging and previous studies have demonstrated similar performance in CT myelography and MR myelography, we chose to combine these data.

\section{Conclusions}

Neither EDX nor imaging is as accurate as previously portrayed. The ideal workup of patients with NBPP likely requires multiple modalities, as the strengths of each test often make up for the other's weaknesses. In the specific setting of identifying lower nerve root avulsions, EDX outperforms imaging. Given that NBPP with lower plexus avulsions is a generally accepted indication for early intervention, EDX can help to identify this pathology in the preoperative setting.

\section{References}

1. Bager B: Perinatally acquired brachial plexus palsy-a persisting challenge. Acta Paediatr 86:1214-1219, 1997

2. Bertelli JA, Ghizoni MF: The towel test: a useful technique for the clinical and electromyographic evaluation of obstetric brachial plexus palsy. J Hand Surg Br 29:155-158, 2004

3. Bisinella GL, Birch R, Smith SJM: Neurophysiological prediction of outcome in obstetric lesions of the brachial plexus. J Hand Surg Br 28:148-152, 2003

4. Borschel GH, Clarke HM: Obstetrical brachial plexus palsy. Plast Reconstr Surg 124 (1 Suppl):144e-155e, 2009

5. Chow BC, Blaser S, Clarke HM: Predictive value of computed tomographic myelography in obstetrical brachial plexus palsy. Plast Reconstr Surg 106:971-979, 2000

6. Foad SL, Mehlman CT, Ying J: The epidemiology of neonatal brachial plexus palsy in the United States. J Bone Joint Surg Am 90:1258-1264, 2008

7. Haerle M, Gilbert A: Management of complete obstetric brachial plexus lesions. J Pediatr Orthop 24:194-200, 2004

8. Malessy MJA, Pondaag W: Nerve surgery for neonatal brachial plexus palsy. J Pediatr Rehabil Med 4:141-148, 2011

9. Malessy MJA, Pondaag W, van Dijk JG: Electromyography, nerve action potential, and compound motor action potentials in obstetric brachial plexus lesions: validation in the absence of a "gold standard". Neurosurgery 65 (4 Suppl):A153A159, 2009

10. Pondaag W, Malessy MJA: Recovery of hand function following nerve grafting and transfer in obstetric brachial plexus lesions. J Neurosurg 105 (1 Suppl):33-40, 2006

11. Pondaag W, Malessy MJ, van Dijk JG, Thomeer RT: Natural history of obstetric brachial plexus palsy: a systematic review. Dev Med Child Neurol 46:138-144, 2004

12. Somashekar D, Yang LJS, Ibrahim M, Parmar HA: High-resolution MRI evaluation of neonatal brachial plexus palsy: A promising alternative to traditional CT myelography. AJNR Am J Neuroradiol 35:1209-1213, 2014

13. Somashekar DK, Wilson TJ, DiPietro MA, Joseph JR, Ibrahim M, Yang LJS, et al: The current role of diagnostic imaging in the preoperative workup for refractory neonatal brachial plexus palsy. Childs Nerv Syst 32:1393-1397, 2016

14. Steens SCA, Pondaag W, Malessy MJA, Verbist BM: Obstetric brachial plexus lesions: CT myelography. Radiology 259:508-515, 2011 
15. Terzis JK, Novikov ML: Radiological and electrophysiological detection of nerve roots avulsion in patients with birth-related brachial plexus paralysis. Semin Plast Surg 19:24-41, 2005

16. Vanderhave KL, Bovid K, Alpert H, Chang KWC, Quint DJ, Leonard JA Jr, et al: Utility of electrodiagnostic testing and computed tomography myelography in the preoperative evaluation of neonatal brachial plexus palsy. J Neurosurg Pediatr 9:283-289, 2012

17. van Dijk JG, Malessy MJ, Stegeman DF: Why is the electromyogram in obstetric brachial plexus lesions overly optimistic? Muscle Nerve 21:260-261, 1998

18. Wade RG, Itte V, Rankine JJ, Ridgway JP, Bourke G: The diagnostic accuracy of $1.5 \mathrm{~T}$ magnetic resonance imaging for detecting root avulsions in traumatic adult brachial plexus injuries. J Hand Surg Eur Vol 43:250-258, 2018

19. Wilson TJ, Chang KWC, Yang LJS: Prediction algorithm for surgical intervention in neonatal brachial plexus palsy. Neurosurgery 82:335-342, 2018

\section{Disclosures}

The authors report no conflict of interest concerning the materials or methods used in this study or the findings specified in this paper.

\section{Author Contributions}

Conception and design: Spires, Chang, Yang. Acquisition of data: Spires, Chang, Yang. Analysis and interpretation of data: all authors. Drafting the article: all authors. Critically revising the article: all authors. Reviewed submitted version of manuscript: all authors. Approved the final version of the manuscript on behalf of all authors: Spires. Statistical analysis: Chang. Administrative/ technical/material support: Chang. Study supervision: Spires, Yang.

\section{Correspondence}

Mary Catherine Spires: University of Michigan, Ann Arbor, MI. mcspires@med.umich.edu. 